\title{
Educational Interventions to Improve Recognition of Delirium: A Systematic Review
}

\author{
Mamata Yanamadala, MBBS, MSc, * Darryl Wieland, PhD, MPH, ${ }^{\dagger+}$ and Mitchell T. Heflin, MD, MHS*
}

Delirium is a common and serious condition that is underrecognized in older adults in a variety of healthcare settings. It is poorly recognized because of deficiencies in provider knowledge and its atypical presentation. Early recognition of delirium is warranted to better manage the disease and prevent the adverse outcomes associated with it. The purpose of this article is to review the literature concerning educational interventions focusing on recognition of delirium. The Medline and Cumulative Index to Nursing and Allied Health Literature (CINHAL) databases were searched for studies with specific educational focus in the recognition of delirium, and 26 studies with various designs were identified. The types of interventions used were classified according to the Predisposing, Reinforcing and Enabling Constructs in Educational Diagnosis and Evaluation (PRECEDE) model, and outcomes were sorted according to Kirkpatrick's hierarchy. Educational strategies combining predisposing, enabling, and reinforcing factors achieved better results than strategies that included one or two of these components. Studies using predisposing, enabling, and reinforcing strategies together were more often effective in producing changes in staff behavior and participant outcomes. Based on this review, improvements in knowledge and skill alone seem insufficient to favorably influence recognition of delirium. Educational interventions to recognize delirium are most effective when formal teaching is interactive and is combined with strategies including engaging leadership and using clinical pathways and assessment tools. The goal of the current study was to systematically review the published literature to determine the effect of educational interventions on recognition of delirium. J Am Geriatr Soc 61:1983-1993, 2013.

Key words: delirium; recognition; education

From the *Duke University Medical Center, Durham, North Carolina; ${ }^{\dagger}$ Geriatrics Services, Palmetto Health Richland, Columbia, South Carolina; and Division of Geriatrics, School of Medicine, University of South Carolina, Columbia, South Carolina.

Address correspondence to Mamata Yanamadala, Division of Geriatrics, Duke University Medical Center, DUMC Box 3003, Durham, NC 27710. E-mail: mamata.yanamadala@duke.edu

DOI: 10.1111 /jgs.12522
$\mathrm{D}$ elirium is defined as the acute onset of altered mental status associated with difficulty sustaining attention and a fluctuating course. It is a serious and common medical condition, particularly in older adults, with high incidence rates in various healthcare settings, including during hospitalization $(6-56 \%)$, postoperatively $(15-53 \%)$, in intensive care $(70-87 \%)$, in post-acute care settings (up to $60 \%$ ), and at the end of life (up to $83 \%$ ). ${ }^{1}$ Delirium is associated with risk of mortality, institutionalization, and worsening or incident dementia. ${ }^{2}$

Despite a growing awareness of its high prevalence and toxicity and availability of effective diagnostic tools, healthcare providers often do not recognize delirium, with nondetection rates as high as $69 \%{ }^{3,4} \mathrm{~A}$ recent study found that delirium remains underrecognized even when a standardized delirium assessment tool is used. ${ }^{5}$ A combination of an unpredictable and atypical presentation and a lack of knowledge make it challenging for providers to recognize delirium. The provider's sense of futility-that is, a perceived lack of available interventions even if delirium is recognized-may impede recognition. The high incidence of delirium in older adults coupled with low rates of recognition contribute to high rates of negative outcomes, including falls, pressure ulcers, long length of stay, healthcare costs, institutionalization, and mortality. ${ }^{4}$ Early recognition of delirium is important not only because of its potential reversibility, but also because diagnosis and treatment of the underlying cause increase the likelihood of preventing negative outcomes. ${ }^{6}$

Previous studies have demonstrated that delirium is often not recognized because of insufficient knowledge and minimal educational emphasis in medical and nursing schools. ${ }^{7}$ In prior studies, $75 \%$ of the nurses interviewed stated that, even after they received formal education on the topic of delirium, they could not differentiate between delirium and dementia. ${ }^{8}$ Although education might play an important role in boosting delirium recognition, the optimal strategies to improve recognition and their usefulness are currently unclear, and several authors have pointed out the limitations of standard delirium teaching methods. ${ }^{9}$ The purpose of this study was to systematically review published delirium education literature to determine the 
effect of educational interventions on learning and patient care outcomes in delirium recognition.

\section{METHODS}

A review was conducted on the Medline and Cumulative Index to Nursing and Allied Health Literature (CINHAL) databases through September 2012 to identify published articles in English. The search combined the terms "delirium," "diagnosis," "diagnosed," "diagnosing," "detection," "detect," “detects," “recognized," "recognised," "recognizing," "recognising," "recognize," "recognise," "recognizes," "recognises," “identify," “identifying," "identified," "identifies," "screen," "screening," "screens," "psychiatric status rating scales," "mass screening," "diagnosis" OR "diagnosis"[Subheading], "delirium/diagnosis," OR "delirium/prevention and control," "curriculum," "pilot projects," "training," "train," "trains," "trained," "educational," "education," “intervention," "staff development," "program," "programs," "education."

The initial search identified 906 abstracts. The screening process is summarized in Figure 1. Abstracts were screened to identify studies describing educational interventions and outcomes focusing on the recognition of delirium. Disagreements regarding classification of 14 abstracts were resolved in discussion between the authors. References from all of the included abstracts were screened manually, and 13 additional pertinent abstracts were identified. Sixty-five abstracts was identified for full-text review and were selected for further analysis if they satisfied the following criteria: primary focus is an educational intervention for recognition of delirium and reported outcomes of the intervention on recognition of delirium. Twenty-six articles were identified for inclusion in this review.

On full-text review, studies were classified according to target audience, setting, and educational intervention. Interventions were classified according to the Predisposing, Reinforcing and Enabling Constructs in Educational Diagnosis and Evaluation (PRECEDE) model (Table 1). ${ }^{10,11} \mathrm{In}$ this model, originally used for studies of continuing medical education, interventions are classified into one of four types: Type 1-predisposing factors (dissemination of information, communication and didactics); Type 2-predisposing factors and enabling strategies (facilitate desired change in performance, for example, by using protocols and guidelines and providing resources); Type 3-predis-

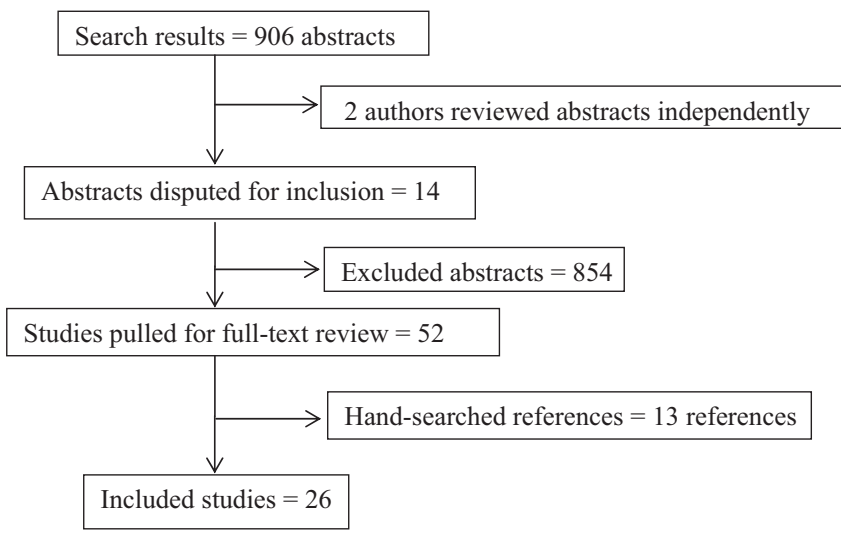

Figure 1. Flow diagram for study inclusion.

\begin{tabular}{lll}
\hline Table 1 & PRECEDE* & Model \\
\hline Type & Factors & \multicolumn{1}{c}{ Strategies } \\
\hline 1 & Predisposing & $\begin{array}{l}\text { Include dissemination of information, } \\
\text { communication, and didactics }\end{array}$ \\
2 & $\begin{array}{l}\text { Predisposing } \\
\text { and enabling }\end{array}$ & $\begin{array}{l}\text { Enabling factors include providing } \\
\text { resources, facilitating desired change in } \\
\text { performance, for example by using } \\
\text { protocols and guidelines }\end{array}$ \\
3 & $\begin{array}{l}\text { Predisposing } \\
\text { and reinforcing } \\
\text { learning through reminders and feedback } \\
\text { from peers and experts }\end{array}$ \\
4 & $\begin{array}{l}\text { Combination of } \\
\text { all three factors }\end{array}$ & $\begin{array}{l}\text { Include a single multifaceted intervention } \\
\text { or a combination of interventions across } \\
\text { all three types }\end{array}$ \\
\hline
\end{tabular}

* Predisposing, Reinforcing and Enabling Constructs in Educational Diagnosis and Evaluation.

posing and reinforcing factors (consolidate learning through reminders and feedback from peers and experts); and Type 4-a single multifaceted intervention or a combination of interventions across all three types. Finally, study results were assigned to one of the four levels of the widely used Kirkpatrick model ${ }^{12}$ (Table 2) to classify the outcomes.

\section{RESULTS}

Of 26 studies identified, 15 were conducted in the United States; three in the United Kingdom; three in Australia; and one each in Canada, Belgium, Italy, Japan, and the Netherlands. Study venues included clinical and educational settings. Thirteen studies targeted staff in the hospital wards, seven in the intensive care unit (ICU), two in long-term care and rehabilitation centers, and four in other settings (course workshops, continuing education, simulation laboratory). Educational interventions targeted nurses in 15 studies, physicians in four studies, and interprofessional staff in seven studies.

\section{Educational Interventions}

Nine studies were classified as Type $1,{ }^{5,9,13,16-21}$ or using a predisposing approach alone, 11 as Type $2,^{14,15,22-30}$ one as Type $3,^{7}$ and the remaining five as Type $4 .{ }^{31-35}$ Most of the Type 4 studies used techniques of the first three study types. An evaluation of the studies and a comparison of their findings are shown in Table 3.

\begin{tabular}{lll}
\hline \multicolumn{2}{c}{ Table } & \multicolumn{2}{c}{ 2. } & Kirkpatrick Model of Learning Outcomes \\
\hline Level & \multicolumn{1}{c}{ Evaluation } & \multicolumn{1}{c}{ Example } \\
\hline 1 & $\begin{array}{l}\text { Reaction_-Participant } \\
\text { satisfaction }\end{array}$ & $\begin{array}{l}\text { Learners' impression of } \\
\text { the teaching experience }\end{array}$ \\
2 & $\begin{array}{l}\text { Learning Staff knowledge } \\
\text { and attitudes }\end{array}$ & $\begin{array}{l}\text { Improved level of } \\
\text { understanding of the } \\
\text { subject area }\end{array}$ \\
3 & $\begin{array}{l}\text { Behavior-Changes in } \\
\text { staff practice }\end{array}$ & $\begin{array}{l}\text { Demonstrable change in } \\
\text { profesional practice }\end{array}$ \\
4 & Results -Changes in & $\begin{array}{l}\text { Significant changes in } \\
\text { health outcome }\end{array}$ \\
\hline
\end{tabular}




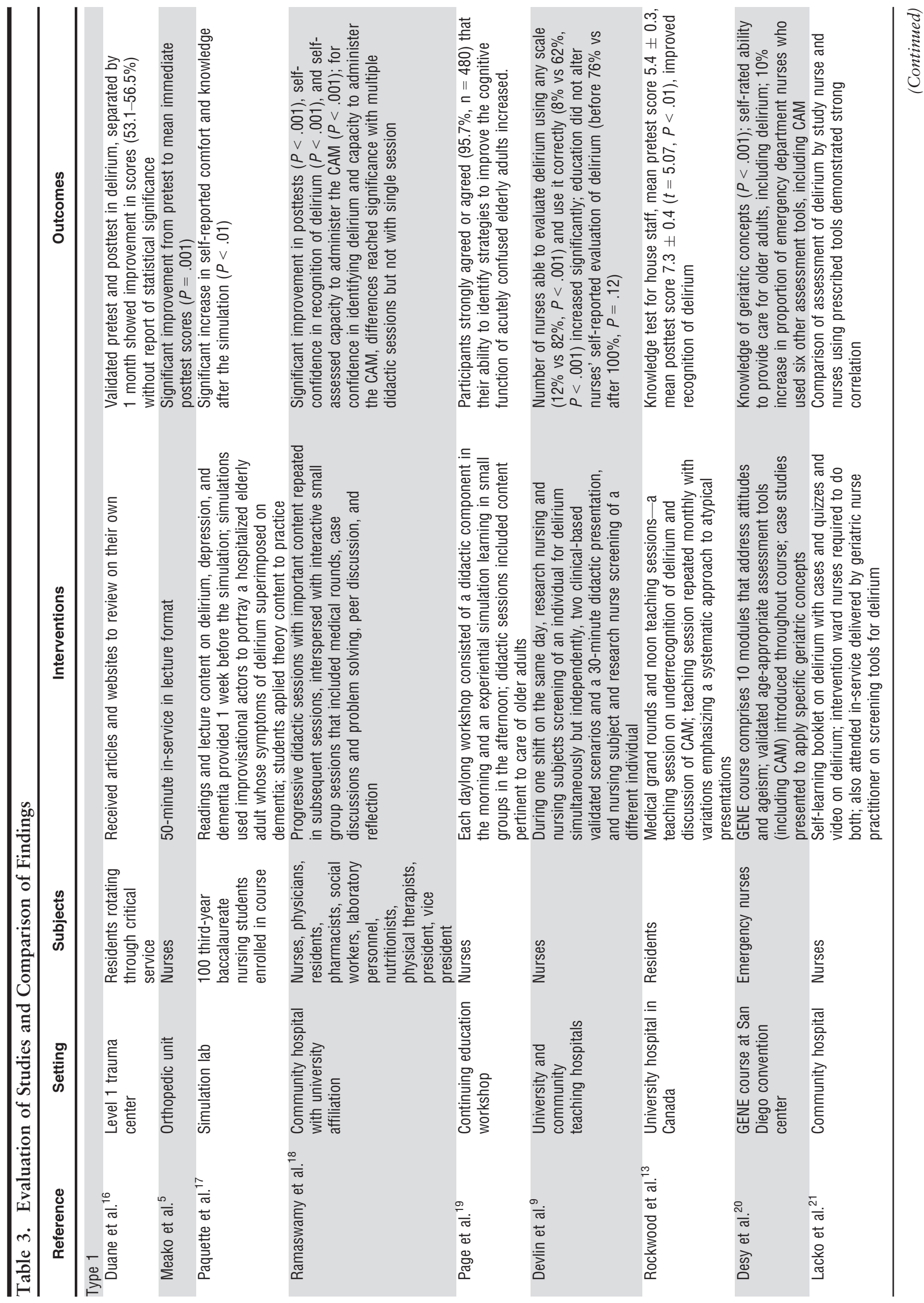




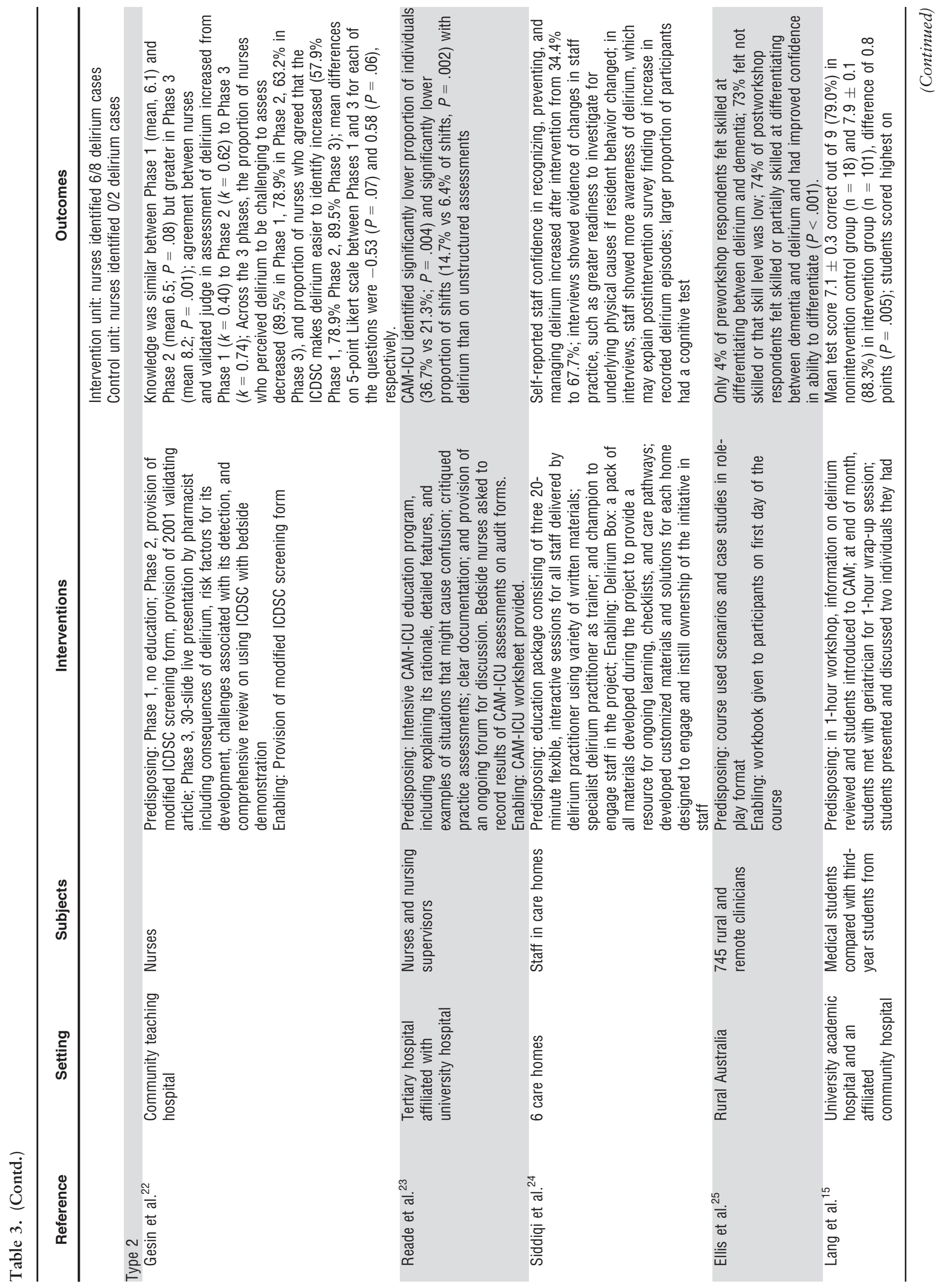




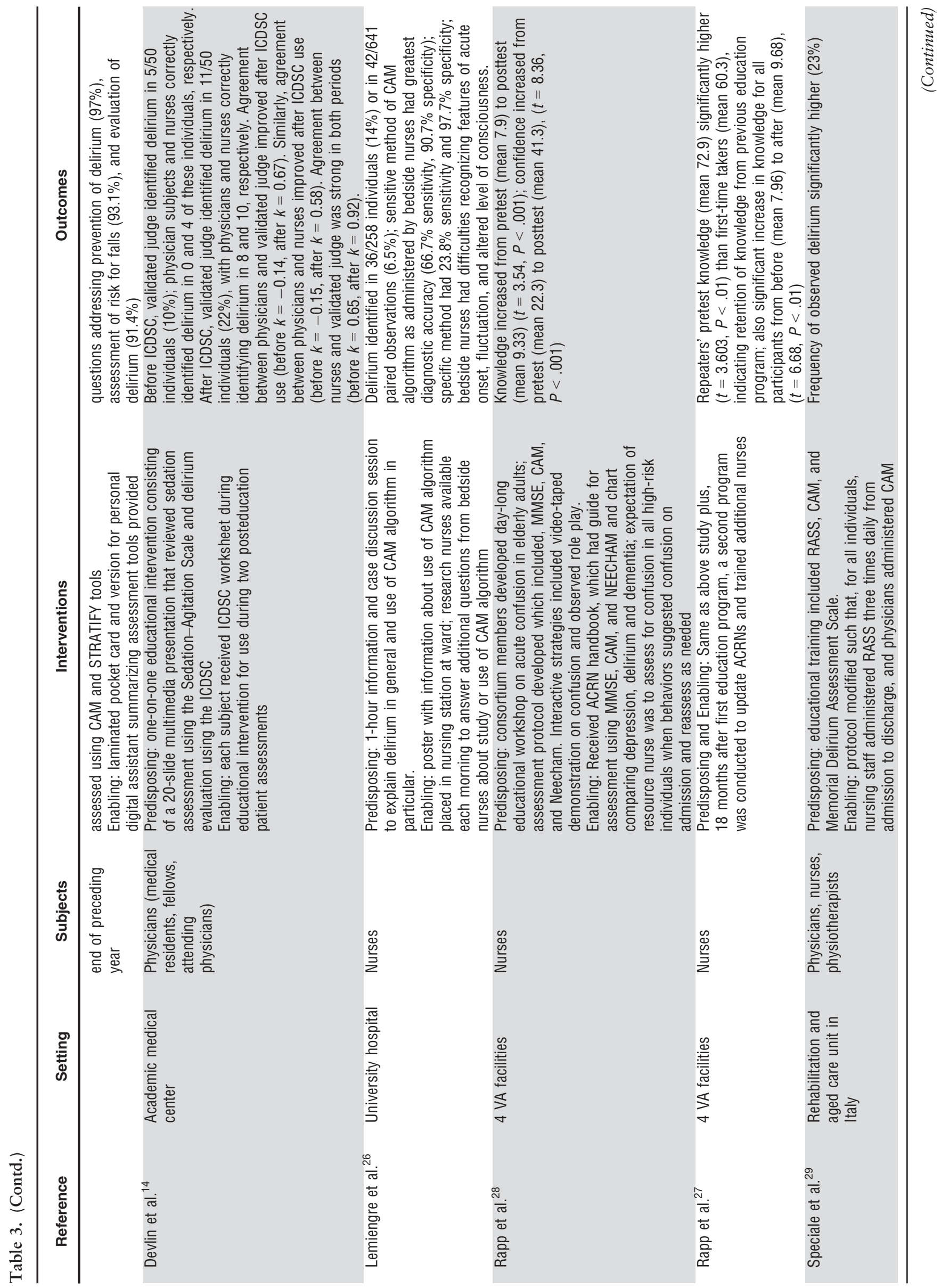




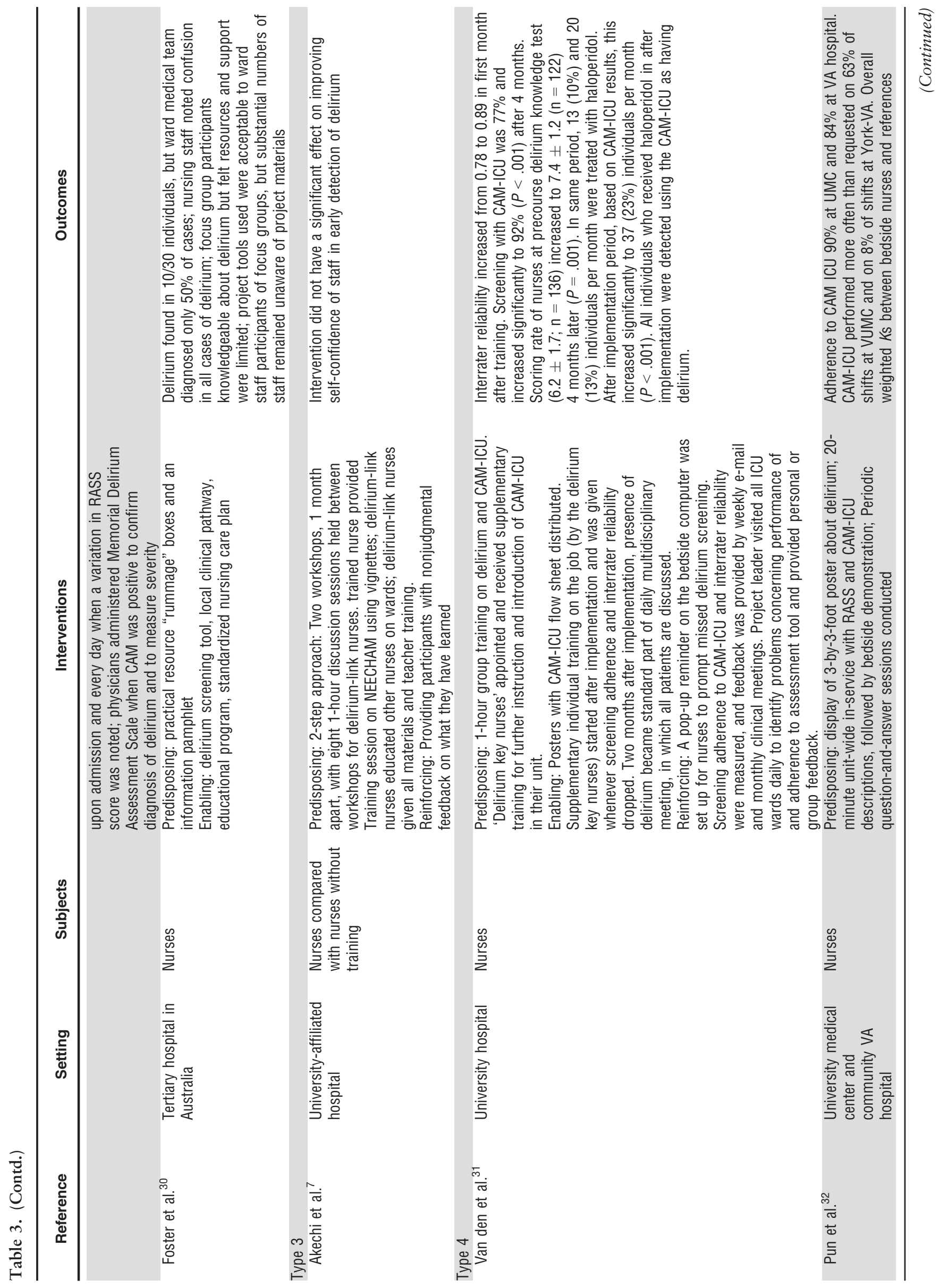




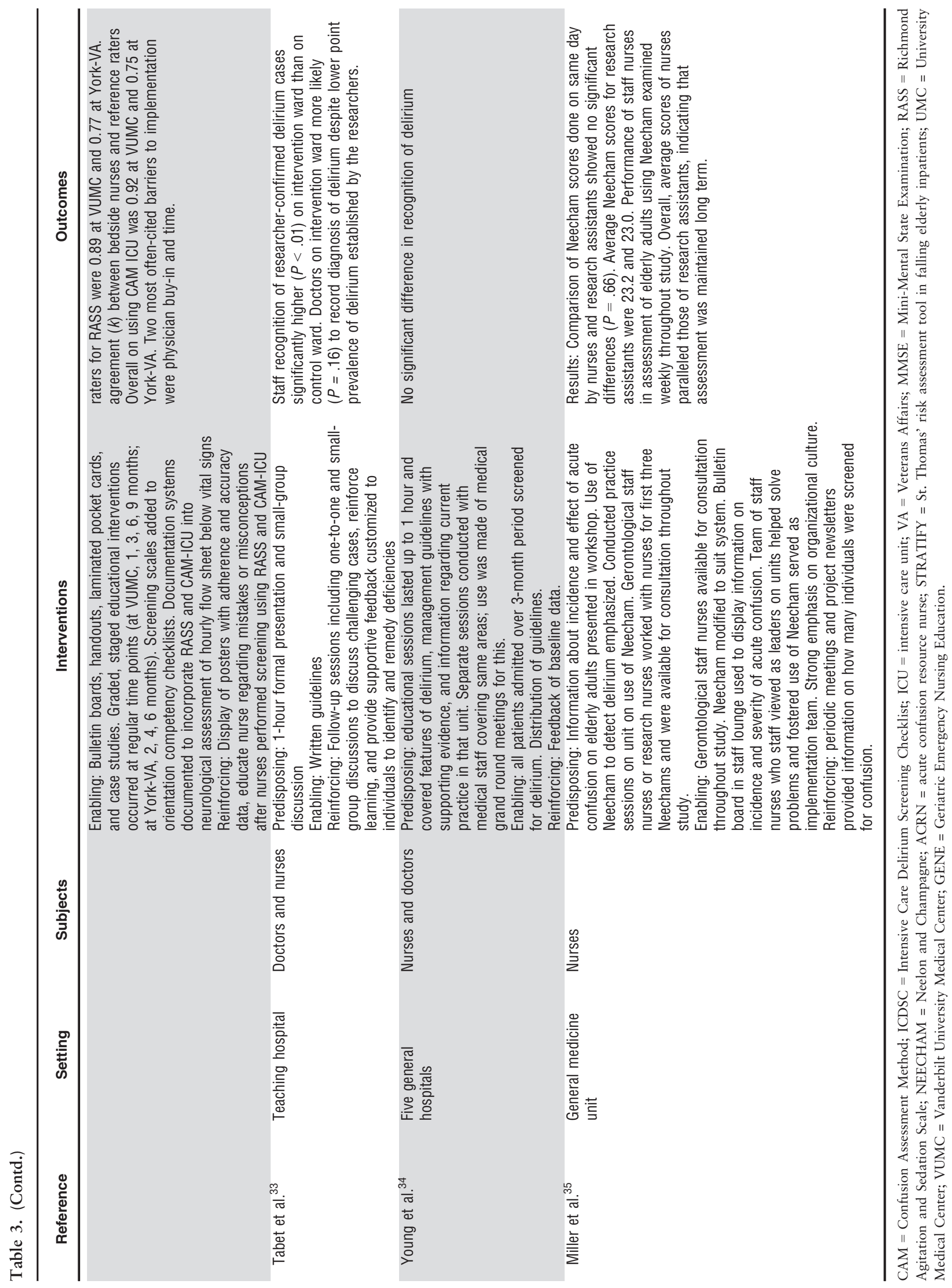


Type 1 interventions included distributing learning material for self-review by learners, ${ }^{16,21}$ lectures $5,13,17,20$ and didactics paired with active learning strategies such as small group case discussions, ${ }^{18}$ simulation, ${ }^{19}$ and script concordance. ${ }^{9}$ Education programs all covered delirium, with a focus on recognition of delirium in all except three studies that focused on teaching broader geriatric content including delirium. ${ }^{16,19,20}$ The target clinicians were multidisciplinary in one study, ${ }^{18}$ nurses alone in six studies, ${ }^{5,9,14,17,19-21}$ and residents alone in two studies. ${ }^{13,16}$ One study reported beneficial patient outcomes (Kirkpatrick Level 4) in terms of greater recognition of delirium after the intervention. ${ }^{21}$ One study reported change in behavior (Kirkpatrick Level 3), with more nurses evaluating for delirium after the intervention. Six of the 10 studies reported knowledge gain (Kirkpatrick Level 2), with four having significant knowledge gain ${ }^{5,9,13,18}$ and two not mentioning whether the knowledge gain was significant. $^{16,21}$ Four of the Type 1 studies reported gain in selfconfidence (Kirkpatrick Level 2) in recognizing delirium, with one study showing significant change ${ }^{18}$ and the others not reporting whether the change was significant. ${ }^{17,19,20}$ Overall, the results of the Type 1 studies showed greater knowledge and self-confidence but with a high degree of variability in reporting the results.

The Type 2 studies used education in conjunction with enabling factors such as Intensive Care Delirium Screening Checklist (ICDSC) forms, ${ }^{14,22}$ the Confusion Assessment Method (CAM) tool, 23,26 protocols and pathways for recognition of delirium, ${ }^{24,29,30}$ trained champions to answer questions, ${ }^{24,26}$ and additional resources such as work books, laminated pocket cards and assessment tools on Personal Digital Assistants (PDA). ${ }^{15,24,25,27,28}$ Six studies included training nurses, ${ }^{22,23,26-28,30}$ one included medical students, ${ }^{15}$ one included physicians, ${ }^{14}$ and three included interdisciplinary staff. ${ }^{24,25,29}$ Two reported positive patient outcomes (Kirkpatrick Level 4) in terms of increased recognition of delirium. ${ }^{29,30}$ Indicators of improved staff performance included increase in recorded delirium and increase in percentage of participants that had an assessment test (Kirkpatrick Level 3), ${ }^{24}$ significant knowledge gain (Kirkpatrick Level 2), ${ }^{14,15,22,27,28}$ and significant increase in self-confidence of recognizing delirium. Overall, many of the Type 2 studies demonstrated significant knowledge gain and improved confidence in recognizing delirium, and some demonstrated behavior modification in identifying delirium and improved recognition of delirium.

The single study identified in the Type 3 category used several workshops to provide information and training on the Neelon and Champagne (NEECHAM) assessment tool to a few nurses. The trained nurses taught additional nurses and provided feedback to the nurses on their performance. This study did not demonstrate any improvement in self-confidence of the nurses in recognizing delirium.

All the Type 4 studies combined predisposing with enabling and reinforcing factors. The educational interventions were targeted to nurses in three studies ${ }^{31,32,35}$ and included interdisciplinary staff in two studies. ${ }^{33,34}$ All studies used enabling strategies in the form of flow sheets, ${ }^{31}$ champions, ${ }^{31,35}$ handouts and pocket cards, ${ }^{32}$ and guidelines. ${ }^{33,34}$ Reinforcing strategies used were feedback on performance by all five studies, reminders, ${ }^{31}$ modification of the current system to incorporate routine assessment, ${ }^{32,34}$ and provision of CAM- ICU ${ }^{32}$ and NEE$\mathrm{CHAM}^{35}$ assessment tools. Two studies demonstrated a significant increase in recognition of delirium (Kirkpatrick Level 4$)^{31,33}$ and two showed improvement in use of an assessment tool (CAM-ICU) to screen for delirium (Kirkpatrick Level 3$)^{31,32,35}$ and significant improvement in knowledge $^{31}$ and interrater agreement on scores on the assessment tools administered. ${ }^{31,32}$ One study reported no change in recognition of delirium. ${ }^{34}$

\section{DISCUSSION}

The literature evaluating the effect of educational interventions on recognition of delirium was reviewed. Studies were classified according to type of educational approach ${ }^{10}$ and evaluated in terms of outcomes for patients and changes in staff performance. Given the challenges that the complex nature of delirium poses, it was not surprising to find that Type 4 studies that combined predisposing, enabling, and reinforcing factors achieved better results than strategies that included one or two of these components (Figure 2). Effective enabling and reinforcing strategies included the use of resource nurses or champions, ${ }^{24,31}$ feedback on performance, ${ }^{31,33}$ and protocols. ${ }^{24,29,31,32}$ Resource nurses or champions in the studies were often trained exclusively to gain expertise in delirium recognition, further train other staff, and act as resources to answer questions and provide feedback on performance. Considering methodological variations, the Type 4 studies, with one exception, showed improved staff performance and recognition of delirium and adherence to protocols when reported.

Type 1 studies involved didactic teaching sessions or workshops and varied widely in interventions and measuring outcomes. Few used active learning strategies such as small group discussions, ${ }^{18}$ simulation, ${ }^{19}$ and script concordance, ${ }^{9}$ although interactive techniques such as case discussions and practical sessions were proven to be more effective in changing outcomes in the literature. ${ }^{11}$ Most Type 1 studies did not report clinical performance and patient care outcomes but reported improved knowledge and self-confidence in recognizing delirium, except two that reported improved recognition of delirium ${ }^{21}$ and behavior modification. ${ }^{9}$ Expectations for measurable effect on clinical outcomes in these studies is low, with the literature showing that isolated educational interventions involving predisposing factors alone are not effective in improving healthcare outcomes or changing provider behavior. $^{36-38}$

Type 2 studies typically involved education sessions with assessment tools and protocols for practice. Most did not report patient care outcomes and change in practice behavior, but many showed significant gain in knowledge or self-confidence, one showed change in behavior, ${ }^{24}$ and two showed improved recognition of delirium. ${ }^{24,29}$ Sequenced sessions have also been shown to have greater effect. $^{22,27}$

Although some studies used validated delirium assessment tools in education and clinical protocols, variations in methodology and evaluation techniques prevented 


\section{Effect of PRECEDE Type on Kirkpatrick Outcomes}

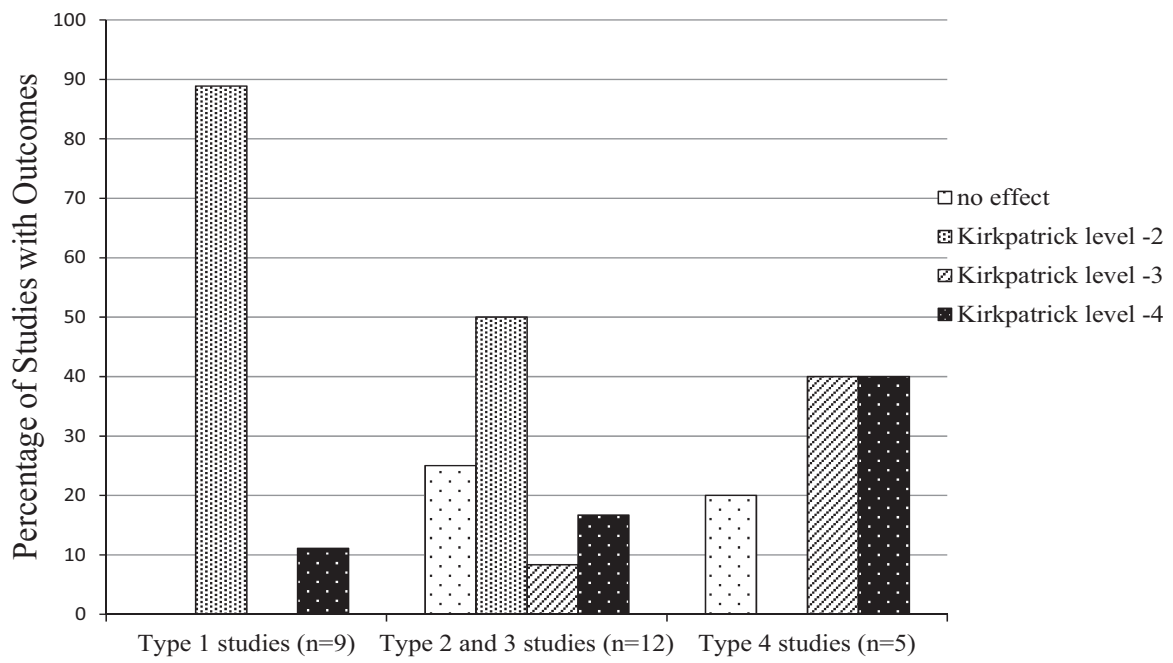

Figure 2. Effect of PRECEDE type interventions on Kirkpatrick level outcomes.

strong conclusions about their effectiveness from being made. Nonetheless, assessment tools appeared to be helpful in knowledge gain and practice change and improved recognition of delirium as seen in prior studies, confirming prior findings that the majority of individuals with delirium are missed when an assessment tool is not used. ${ }^{31}$

In this review educational interventions to recognize delirium were classified according to factors that can facilitate learning and change practice and patient outcomes. A similar approach has recently been used in two other reviews $^{38,39}$ to understand educational interventions in the prevention and management of delirium. Both reviews concluded that the most effective delirium education programs were multifaceted and comprehensive and included enabling and reinforcing techniques in addition to knowledge transmission. This is the only review to the knowledge of the authors that has focused on educational interventions aiming to improve recognition of delirium. Some argue that efforts at delirium recognition are futile until appropriate treatments are identified, ${ }^{32}$ but in certain studies, authors identified providers who felt that awareness of delirium improved the care they delivered despite the absence of abundant evidence-based delirium prevention and treatment options. ${ }^{32}$

\section{Limitations}

Inclusion of studies with various approaches to education and evaluation presented difficulty in combining results and drawing strong conclusions. Also, it was not possible to include unpublished studies in this review, increasing the potential for publication bias. Some studies that involved improved recognition of delirium were excluded because of inadequate description of their educational interventions. ${ }^{40,41}$ Nonetheless, the search was comprehensive, and the studies identified represented the best evidence available to answer the question.

An adaptation of the PRECEDE model was used to study the effectiveness of continuing medical education for physicians, but it is not known whether the same classifi- cation can be applied to most of the studies in this review that focus on interventions for nurses and allied health professionals. This point is significant because most of the interventions focused on nonphysician professionals, but use of the PRECEDE model has strong precedent in this type of review and provides an effective framework for characterizing educational interventions. ${ }^{38,39}$

\section{Recommendations for Research}

The heterogeneity of educational methodologies in the articles included in this review made it hard to draw strong conclusions on what interventions would be effective in recognizing delirium. In this review and the others that focused on managing delirium, comprehensive interventions proved to be more effective for all four Kirkpatrick levels of outcomes. These results are consistent with education literature, which revealed that comprehensive multifaceted strategies including reinforcing and enabling approaches were more effective in improving patient outcomes ${ }^{18,31,36,37}$ than purely didactic experiences, which can improve competence in knowledge, skills, and attitudes but are less likely to bring behavior change or improved outcomes. ${ }^{18}$

Methods of evaluation fell short in using validated tools for evaluation of knowledge gain, practice change, and patient outcomes in some of the of the studies included in this review. Studies often used self-evaluation of performance without any objective measurements to confirm behavior change. Future studies should therefore be designed to develop and validate strong evaluation techniques focusing on all Kirkpatrick levels, including endpoints such as patient outcomes, cost-effectiveness, and time efficiency.

It is likely that following some basic educational principles $^{42}$ would enhance the effect of many of these programs, including making the education programs relevant to the perceived needs of the participants, using teaching methods that emphasize the integration of new information into what is already known, and providing partici- 
pants with nonjudgmental feedback on what they have learned. Most of these principles were achievable in some of the studies through the use of nurse champions because they were able to interact on a personal level and had an understanding of the needs. ${ }^{24,31}$ One illustrative example from this review ${ }^{24}$ demonstrated improvement in outcomes at all Kirkpatrick levels by recruiting working groups from staff who volunteered to participate, and these groups identified important questions on delirium relevant to their setting and produced a variety of resources and customized solutions for each setting.

Case studies, which are an integral part of education in health care, give learners the opportunity to consider multiple facets of a clinical situation, to expand their knowledge base, and to develop problem-solving and critical-thinking skills. ${ }^{19}$ Simulation and script concordance techniques use case studies and are well supported in learning theory to train healthcare providers as it promotes active engagement in thinking, analyzing, acquiring in-depth understanding, and applying knowledge. ${ }^{9,17,19}$ These techniques may prove especially effective for assessing complex clinical conditions such as delirium because, in an unfolding case study, learners can experience the clinical situation as it progresses over time; they are exposed to the full context and complexity of the evolving setting and disease progression. ${ }^{19}$

Educational interventions should be implemented with buy-in from leadership because the success of the intervention depends on administrative understanding, belief, and support. In several cases from this review, hospital leadership was vital in promoting and sustaining educational programs directed at improving outcomes. ${ }^{18}$ One study indicated that senior management support (or lack thereof) was viewed as the most important factor determining whether their intervention program was successfully implemented and sustained. ${ }^{18}$

Assessment for delirium appears to be most effective if clinicians are trained in the use of standardized tools, because the ability to identify delirium improves when a validated delirium assessment scale is used. ${ }^{9}$ Using validated assessment tools as part of the interventions should be feasible because the mean assessment time with most of the validated tools is 2 to 5 minutes. ${ }^{31}$ Although optimal adherence to assessment is not known, some of the studies used $80 \%$ adherence as acceptable. ${ }^{31}$

\section{CONCLUSION}

Improving the recognition of delirium in older adults is a complex problem requiring a sophisticated solution. Many experts argue that the recognition of delirium is the fundamental obstacle to better care of individuals with delirium. ${ }^{43}$ Educational interventions designed to improve recognition of delirium by increasing knowledge of delirium and skills in methods of its detection are important but inadequate. Educational interventions to recognize delirium are most effective when formal teaching is interactive and combined with enabling and reinforcing strategies. Effective strategies include providing feedback, clinical pathways, and reminders combined with smallgroup or individual case-based discussions. The role of champions appears to help with outcomes. Valuable insights from health education literature on how clinicians learn should be used to identify effective interventions when designing programs to enhance efforts in improving delirium recognition.

\section{ACKNOWLEDGMENTS}

Conflict of Interest: The editor in chief has reviewed the conflict of interest checklist provided by the authors and has determined that the authors have no financial or any other kind of personal conflicts with this paper.

Author Contributions: Yanamadala: literature search, screening articles for inclusion, analysis of studies, preparation of manuscript. Heflin: screening articles for inclusion, design, writing of manuscript. Wieland: design of study, critical evaluation and revision of manuscript.

\section{Sponsor's Role: None.}

\section{REFERENCES}

1. Inouye SK. Delirium in older persons. N Engl J Med 2006;354:1157-1165.

2. Witlox J, Eurelings LSM, de Jonghe JFM et al. Delirium in elderly patients and the risk of postdischarge mortality, institutionalization, and dementia. JAMA 2010;304:443-451.

3. Vollmer C, Bond J, Eden B et al. Incidence, prevalence, and under-recognition of delirium in urology patients. Urol Nurs 2010;30:235-241.

4. Greer N, Rossom R, Anderson P et al. Delirium: Screening, Prevention, and Diagnosis-A Systematic Review of the Evidence [on-line]. Washington, DC: Department of Veterans Affairs, 2011. Available at http://www.ncbi. nlm.nih.gov/books/NBK82554/ Accessed May 15, 2013.

5. Meako ME, Thompson HJ, Cochrane BB. Orthopaedic nurses' knowledge of delirium in older hospitalized patients. Orthop Nurs 2011;30:241-248.

6. Schuurmans MJ, Duursma SA, Shortridge-Baggett LM. Early recognition of delirium: review of the literature. J Clin Nurs 2001;10:721-729.

7. Akechi $\mathrm{T}$, Ishiguro $\mathrm{C}$, Okuyama $\mathrm{T}$ et al. Delirium training program for nurses. Psychosomatics 2010;51:106-111.

8. Fick D, Foreman M. Consequences of not recognizing delirium superimposed on dementia in hospitalized elderly individuals. J Gerontol Nurs 2000;26:30-40.

9. Devlin JW, Marquis F, Riker RR et al. Combined didactic and scenariobased education improves the ability of intensive care unit staff to recognize delirium at the bedside. Crit Care 2008;12:R19.

10. Green LW, Kreuter MW, Deeds SG et al. Health Education Planning: A Diagnostic Approach. Mountain View, CA: Mayfield Publishing Company, 1980.

11. Davis DA, Thomson MA, Oxman AD et al. Evidence for the effectiveness of CME. JAMA 1992;268:1111-1117.

12. Kirkpatrick DL. Evaluating Training Programs: The Four Levels. San Francisco: Berrett-Koehler Publishers, 1994.

13. Rockwood K, Cosway S, Stolee P et al. Increasing the recognition of delirium in elderly patients. J Am Geriatr Soc 1994;42:252-256.

14. Devlin JW, Fong JJ, Schumaker G et al. Use of a validated delirium assessment tool improves the ability of physicians to identify delirium in medical intensive care unit patients. Crit Care Med 2007;35:2721-2724.

15. Lang VJ, Clark NS, Medina-Walpole Al et al. Hazards of hospitalization: hospitalists and geriatricians educating medical students about delirium and falls in geriatric inpatients. Gerontol Geriatr Educ 2008;28:94-104.

16. Duane TM, Fan L, Bohannon A et al. Geriatric education for surgical residents: identifying a major need. Am Surg 2011;77:826-831.

17. Paquette M, Bull M, Wilson S et al. A complex elder care simulation using improvisational actors. Nurse Educ 2010;35:254-258.

18. Ramaswamy R, Dix EF, Drew JE et al. Beyond grand rounds: a comprehensive and sequential intervention to improve identification of delirium. Gerontologist 2011;51:122-131.

19. Page JB, Kowlowitz V, Rhodes Alden K. Development of a scripted unfolding case study focusing on delirium in older adults. J Contin Educ Nurs 2010;41:225-230.

20. Désy PM, Prohaska TR. The Geriatric Emergency Nursing Education (GENE) course: an evaluation. J Emerg Nurs 2008;34:396-402.

21. Lacko LA, Dellasega C, Salerno FA et al. The role of the advanced practice nurse in facilitating a clinical research study: screening for delirium. Clin Nurse Spec 2000;14:110-118. 
22. Gesin G, Russell BB, Lin AP et al. Impact of a delirium screening tool and multifaceted education on nurses' knowledge of delirium and ability to evaluate it correctly. Am J Crit Care 2012;21:e1-e11.

23. Reade MC, Eastwood GM, Peck L et al. Routine use of the Confusion Assessment Method for the Intensive Care Unit (CAM-ICU) by bedside nurses may underdiagnose delirium. Crit Care Resusc 2011;13:217-224.

24. Siddiqi N. Preventing delirium in older people. Nurs Times 2011;107: $22-23$.

25. Ellis I, Philip T. Improving the skills of rural and remote generalists to manage mental health emergencies. Rural Remote Health 2010;10:1503.

26. Lemiengre J, Nelis T, Joosten E et al. Detection of delirium by bedside nurses using the confusion assessment method. J Am Geriatr Soc 2006;54:685-689.

27. Rapp CG, Ongega L, Tripp-Reimer $\mathrm{T}$ et al. Training of acute confusion resource nurses: knowledge, perceived confidence, and role. J Gerontol Nurs 2001;27:34-40.

28. Rapp CG, Onega LL, Tripp-Reimer $\mathrm{T}$ et al. Unit-based acute confusion resource nurse: an educational program to train staff nurses. Gerontologist 1998;38:628-632.

29. Speciale S, Bellelli G, Trabucchi M. Staff training and use of specific protocols for delirium management. J Am Geriatr Soc 2005;53:1445-1446.

30. Foster NM, Waldron NGHD, Donaldson $\mathrm{M}$ et al. A quality improvement project to prevent, detect, and reduce delirium in an acute setting. Austral J Advance Nurs 2011;28:24.

31. Van Den Boogaard M, Pickkers P, Van Der Hoeven H et al. Implementation of a delirium assessment tool in the ICU can influence haloperidol use. Crit Care 2009;13:R131.

32. Pun BT, Gordon SM, Peterson JF et al. Large-scale implementation of sedation and delirium monitoring in the intensive care unit: a report from two medical centers. Crit Care Med 2005;33:1199-1205.
33. Tabet N, Hudson S, Sweeney V et al. An educational intervention can prevent delirium on acute medical wards. Age Ageing 2005;34:152-156.

34. Young L, George J. Do guidelines improve the process and outcomes of care in delirium? Age Ageing 2003;32:525-528.

35. Miller J, Neelon V, Champagne $M$ et al. The assessment of acute confusion as part of nursing care. Appl Nurs Res 1997;10:143-151.

36. Mowatt G, Grimshaw JM, Davis DA et al. Getting evidence into practice: the work of the Cochrane Effective Practice and Organization of Care Group (EPOC). J Contin Educ Health Prof 2001;21:55-60.

37. Grimshaw JM, Shirran L, Thomas $\mathrm{R}$ et al. Changing provider behavior: an overview of systematic reviews of interventions. Med Care 2001;39(8 Suppl 2): II2-II45.

38. Wand APF. Evaluating the effectiveness of educational interventions to prevent delirium. Australas J Ageing 2011;30:175-185.

39. Teodorczuk A, Welfare M, Corbett S et al. Developing effective educational approaches for Liaison Old Age Psychiatry teams: a literature review of the learning needs of hospital staff in relation to managing the confused older patient. Int Psychogeriatr 2010;22:874-885.

40. Gaudreau J-D, Gagnon P, Harel F et al. Impact on delirium detection of using a sensitive instrument integrated into clinical practice. Gen Hosp Psychiatry 2005;27:194-199.

41. Gaudreau J-D, Gagnon P, Harel F et al. Fast, systematic, and continuous delirium assessment in hospitalized patients: the nursing delirium screening scale. J Pain Symptom Manage 2005;29:368-375.

42. Rockwood K. Educational interventions in delirium. Dement Geriatr Cogn Disord 1999;10:426-429.

43. Foreman M, Milisen K. Improving recognition of delirium in the elderly. Primary Psychiatry 2004;11:46-50. 